\title{
EPIS AND MONOS WHICH MUST BE ISOS
}

\author{
DAVID J. FIELDHOUSE
}

\author{
Department of Mathematics and Statistics \\ University of Guelph \\ Guelph, Ontario NIG 2Wl CANADA
}

(Received March 21, 1984)

\begin{abstract}
Orzech [1] has shown that every surjective endomorphism of a noetherian module 18 an isomorphism. Here we prove analogous results for injective endomorphisms of noetherian injective modules, and the duals of these results. We prove that every injective endomorphism, with large 1mage, of a module with the descending chain condition on large submodules 18 an 1somorphism, which dualizes a result of Varadarajan [2]. Finally we prove the following result and its dual: if $p$ is any radical then every surjective endomorphism of a module $M$, with kernel contalned in $\mathrm{pM}$, 1s an 1somorph1sm, provided that every surjective endomorphism of $\mathrm{pM}$ is an 1somorphism.
\end{abstract}

KEY WORDS AND PHRASES. Injective endomorphism, surjective endomorphism, ascending chain condition (ACC), descending chain condition (DCC), artinian module, noetherian module, injective module, projective module, injective hull, projective cover, small submodule, large submodule, preradical, radical, 1dempotent preradical.

1980 AMS MATHEMATICS SUBJECT CLASSIFICATION CODES. 16A21, 16A33, 16A35.

1. INTRODUCTION.

Orzech [1] has shown that every surjective endomorphism of a noetherian 
module is an isomorphism. Here we prove analogous results for injective endomorphisms of noetherian injective modules, and the duals of these results. We prove that every injective endomorphism, with large image, of a module with the descending chain condition on large submodules is an isomorphism, which dualizes a result of Varadarajan [2]. Finally we prove the following result and its dual: if $p$ is any radical then every surfective endomorphism of a module $M$, with kernel contained in $P M$, is an 1somorphism, provided that every surjective endomorphism of $\mathrm{pM}$ is an isomorphism.

2. CONVENTIONS, NOTATION, AND TERMINOLOGY.

Unless otherwise stated, we use the following conventions, notation, and terminology.

All rings are assoclative, but not necessarily commutative. Every ring has a multiplicative identity element, denoted by 1 , which is preserved by ring homomorphisms, inherited by subrings, and acts as the identity operator on modules.

We use the word map for module homomorphism. Maps are written on the side opposite to that of the scalars. Thus the order of writing map compositions depends on the side of the module.

If $M$ and $N$ are $R$-modules we usually write $\operatorname{Hom}(M, N)$ for $\operatorname{Hom}_{R}(M, N)$ when no confusion can arise.

The symbols < and > will be used to denote proper set theoretical inclusion and containment, respectively, as well as the usual order relationships. The symbols < and 2 , respectively, are used for the preceeding if equality can occur.

We recall that a module is noetherian iff it satisfies the ascending chain condition (ACC) for submodules, and artinian iff it satisfies the descending chain condition (DCC) for submodules.

A submodule $L$ of a module $M$ is defined to be large (or essential) iff it has a non-zero intersection with every non-zero submodule of $M$. A map is large iff its image is a large submodule. It is easy to verify that the product of large injective maps 1s large and that under a surjective map preimages of large submodules are large.

Dually, a submodule $S$ of a module $M$ is defined to be small (or superfluous) iff whenever $S+M^{-}=M$ for a submodule $M^{-}$of $M$ then we must have $M^{-}=M$. A map is small iff its kernel is a small submodule. It is easy to verify that the product of small surjective maps 1 s small and that small submodules are small in overmodules.

In the following we shall state a number of results and their duals, but usually only give the proof of one. 


\section{MAIN RESULTS.}

Let us $f i x$ a right $R$-module $M$ and a submodule $M^{-}$, and denote the factor module $M / M^{-}$by $M^{\prime \prime}$.

THEOREM 1 .

(1) If $M^{\prime \prime}$ is artinian then every injective map in $\operatorname{Hom}\left(M, M^{\prime \prime}\right)$ is an 1somorphism.

(2) If $M^{-}$is noetherian then every surjective map in $\operatorname{Hom}\left(M^{-}, M\right)$ is an 1somorphism.

PROOF. Part (2) 1s basically a Theorem of Orzech [1]. For the sake of completeness we shall prove its dual, part (1).

Let $f$ be an infective map in $\operatorname{Hom}\left(M, M^{\prime \prime}\right)$. We define the following descending chain of submodules of $M$ : let $M_{0}=M$, and for $n>0, M_{n+1}$ is defined by $M_{n+1} / M^{-}=f M_{n}$.

One verifies readily that the $M_{n}$ form a descending chain of submodules of $M$, which gives rise to the descending chain $M_{n} / M^{-}$of submodules of $M / M^{-}$, which must terminate since $M^{\prime \prime}$ is artinian. Let $n$ be the least integer such that $M_{n+1} / M^{-}=M_{n} / M^{-}$. If $n=0$ then the map $f$ is surjective, and hence an isomorphism. Otherwise, if $n>0$ then $f M_{n}=f M_{n-1}$, wh1ch is 1mpossible since $M_{n}<M_{n-1}$ and the map $f$ is injective.

We now turn to the dual problems.

THEOREM 2 .

(1) If $M^{\prime \prime}$ is an artinian module and $M$ is a projective module then every surjective map in $\operatorname{Hom}\left(M^{\prime \prime}, M\right)$ is an 1somorphism.

(2) If $M^{-}$is a noetherian module and $M$ is an infective module then every injective map in $\operatorname{Hom}\left(M^{-}, M\right)$ is an 1somorphism.

PROOF. We shall prove only part (1) and leave the proof of this and all subsequent dual proofs to the reader.

Let $f$ be a surjective map in $\operatorname{Hom}\left(M^{\prime \prime}, M\right)$. Since $M 18$ profective this map splits, so that $\mathrm{fg}=1_{M}$, the 1dentity map on $M$, with $\mathrm{g}$ an infective map in Hom $\left(M, M^{\prime \prime}\right)$. The preceeding Theorem implies that $g$ is an 1somorph1sm, and hence $f$ is too.

COROLLARY.

(1) If $M$ is an artinian projective module then every surjective map in $\operatorname{Hom}\left(M^{\prime \prime}, M\right)$ is an 1somorphism.

(2) If $M$ is a noetherian injective module then every injective map in $\operatorname{Hom}\left(M, M^{-}\right)$is an 1somorph1sm.

THEOREM 3.

(1) If $M$ has an artinian projective cover then every surjective map in $\operatorname{Hom}(M, M)$ is an isomorphism.

(2) If $M$ has a noetherian injective hull then every injective map in $\operatorname{Hom}(M, M)$ is an 1somorphism. 
PROOF.

(1) Let $P$ be an artinian projective cover of $M$, with corresponding kernel $K$. Any surjective map $f$ in $\operatorname{Hom}(M, M)$ 1ifts to a map $g$ in $\operatorname{Hom}(P, P)$ which must be surjective since $P$ is a cover. Since $P$ is artinian and projective $g$ must be an 1somorphism, which means that its restriction to $\mathrm{K}$ is injective. But $\mathrm{K}$ is artinian since $P$ is, and therefore the restriction must be an 1somorphism. Hence the map $f$ is an isomorphism.

(2) has a dual proof.

The following theorem gives the dual of and a slight generalization of a result of Varadarajan [2]. The proof we give, and its dual, differ from his.

THEOREM 4.

(1) If $M^{\prime \prime}$ has the descending chain condition on large submodules then every injective map in $\operatorname{Hom}\left(M, M^{\prime \prime}\right)$ with large image is an 1somorphism.

(2) If $M^{-}$has the ascending chain condition on small submodules then every surjective map In $\operatorname{Hom}\left(M^{-}, M\right)$ with small kernel is an 1somorphism.

PROOF.

(1) Let $f$ be an injective map in $\operatorname{Hom}\left(M, M^{\prime \prime}\right)$. As before we define a descending chain of submodules: $M_{0}=M$ and for $n>0, M_{n+1} / M^{-}=f M_{n}$.

In order to use the proof given above, we must verify that we now have a descending chain of large submodules of $M^{\prime \prime}$. We do this by induction. By assumption, for $n=0$ we have $M_{1} / M^{-}=f M$ large in $M^{\prime \prime}$. Assume now that $M_{n} / M^{-}$ is large in $M / M^{-}$. Since under a surjection preimages of large submodules are large, we have $M_{n}$ large in $M$. We need only show that $M_{n+1} / M^{-}$1s large in $M_{n} / M^{-}$and then use the transitivity of largeness. Let $m^{\prime \prime}$ be a non-zero element of $M_{n} / M^{-}=f M_{n-1}$. Since $f$ is injective there exists a unique (nonzero) element $m$ in $M_{n-1}$ such that $f m=m$ ". Since $M_{n}$ is large in $M$ there exists an element $r$ in $R$ such that $r m$ is a non-zero element of $M_{n}$. Since $f$ is injective the element $f(r m)=\mathrm{rm}^{\prime \prime}$ is a non-zero element of $M_{n+1}$, which concludes the proof.

(2) The preceeding proof can be dualized.

COROLLARY .

(1) If $M$ has the descending chain condition on large submodules then every injective map in $\operatorname{Hom}\left(M, M^{\prime \prime}\right)$ with large 1mage is an 1somorphism.

(2) If $M$ has the ascending chain condition on small submodules then every surjective map in $\operatorname{Hom}\left(M^{-}, M\right)$ with small kernel is an 1somorphism. PROOF.

(1) Under a surjective map preimages of large submodules are large.

(2) Small submodules are small in overmodules. 


\section{PRERADICALS.}

For preradicals we generally use the notation and terminology of the book of Stenstrom [3], with some minor modifications. We use the letter p to denote a preradical, and 0 and 1 to denote the zero and identity functors, respectively.

A preradical is defined to be a subfunctor of the identity functor.

Each preradical $p$ defines an ascending chain of preradicals $p_{a}$, indexed by the ordinals, as follows: for the ordinal $a=0: P_{0} M=0$; for non-limit ordinals a $>0: \mathrm{p}_{\mathrm{a}} \mathrm{M} / \mathrm{p}_{\mathrm{a}-1} \mathrm{M}=\mathrm{p}\left(\mathrm{M} / \mathrm{p}_{\mathrm{a}-1} \mathrm{M}\right)$; and for limit ordinals a: $p_{a} M=\sum p_{b} M$ with the sum taken over all ordinals $b<a$.

If we let $p^{-}=\left\{p_{a}\right.$ then we have an ascending chain of preradicals:

$$
0=p_{0}<p=p_{1}<p_{2}<\ldots<p_{a}<\ldots<p^{-}<1 .
$$

Each preradical $p$ also defines a descending chain of preradicals $p^{b}$, Indexed by the ordinals, as follows: for the ordinal $b=0: p^{0} M=M$; for non-1imit ordinals $b>0: p^{b_{M}}=p\left(p^{b-1} M\right)$; and for 11mit ordinals $b, p^{b} 18$ the intersection of all $p^{a_{M}}$ with the intersection taken over $a l 1 a<b$.

If we let $p^{\prime \prime}$ denote the intersection of the $p^{b}$ then we have a descending chain of preradicals :

$$
1=p^{0}>p=p^{1}>p^{2}>\ldots>p^{b}>\ldots>p^{\prime \prime}>0 \text {. }
$$

A preradical $p$ is radical iff $p=p^{-}$, or equivalently, $p(M / p M)=0$ for all modules $M$. For any preradical $p$ we have $\left(p^{-}\right)^{-}=p^{-}$and hence $p^{-}$is radical.

A preradical $p$ is idempotent iff $p(p M)=p M$ for all modules $M$. It 18 easy to verify that for any preradical $p$ the preradical $p^{\prime \prime}$ is idempotent.

For any module $M$ and ordinals $a$ and $b$ let $M_{a}=p_{a} M$ and $M^{b}=p^{b}$. This defines a corresponding ascending chain of submodules:

$$
0=M_{0}<M_{1}<M_{2}<\ldots<M_{a}<\ldots<M
$$

and a corresponding descending chain of submodules:

$$
M=M^{0}>M^{1}>M^{2}>\ldots>M^{b}>\ldots>0 \text {. }
$$

LEMMA. Let $M$ and $N$ be right $R$-modules and $p$ be any preradical. (1) If $M_{a}=M_{a+1}$ and $f$ is a surjective map in $\operatorname{Hom}(M, N)$ with kernel contained In $M_{a}$ then the naturally Induced map $f_{a}$ In $\operatorname{Hom}\left(M_{a}, N_{a}\right)$ is surjective. (2) If $M^{b}=M^{b+1}$ and $f$ is an injective map in $\operatorname{Hom}(N, M)$ with image containing $M^{b}$ then the naturally induced map $f^{b}$ in $\operatorname{Hom}\left(N / N^{b}, M / M^{b}\right)$ is infective.

PROOF.

(1) Since $p_{a}$ is a preradical the image under $f$ of $M_{a}$ is contained in $N_{a}$. Since the kernel of $f$ is contained in $M_{a}$ there is a canonical map from $N$ to $M / M_{a}$, under which the Image of $N_{a}$ is contained in $p_{a}\left(M / M_{a}\right)$, which 1 s zero since $M_{a}=M_{a+1}$. This 1mplies that $N_{a}$ equals the 1mage of $M_{a}$ under $f$, $1 . e$. $f_{a}$ is surjective.

(2) The proof $1 \mathrm{~s}$ dual. 
THEOREM 5. Let $p$ be any preradical.

(1) If $p_{a}=p_{a+1}$ and every surjective map from a submodule of $M_{a}$ to $M_{a}$ Itself is an 1somorphism, then every surjective map in $\operatorname{Hom}\left(M^{-}, M\right)$ with kernel contained in $\mathrm{M}^{-}$a 1 an isomorphism.

(2) If $\mathrm{p}^{\mathrm{b}}=\mathrm{p}^{\mathrm{b}+1}$ and every injective map from $M^{\mathrm{b}}$ itself to a factor module of $M^{b}$ is an 1somorphism, then every injective map in $\operatorname{Hom}\left(M, M^{\prime \prime}\right)$ with Image containing $M^{\prime \prime b}$ is an isomorphism.

PROOF.

(1) If $f$ is a surjective map in $\operatorname{Hom}\left(M^{-}, M\right)$ then the induced map $f_{a}$ in $\operatorname{Hom}\left(M^{-}, M_{a}\right)$ is surjective and hence an 1somorphism since $M^{-}$is a submodule of $M_{a}$. Since the kernel of $f$ is contained in $M^{-}$it must be zero.

(2) The proof is dual.

\section{REFERENCES}

1. ORZECH, M., "Onto Endomorphisms are Isomorph1sms", Amer. Math. Monthly, 78(1971) 357-362.

2. Varadarajan, K., "Modules with Supplements", Pac..J. Math. $\underline{82}$ (1979) 559-564.

3. STENSTROM, Bo., "Rings of Quotients", Springer-Verlag, 1975. 


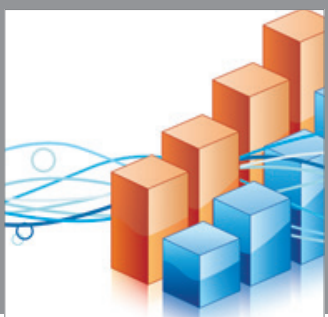

Advances in

Operations Research

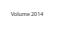

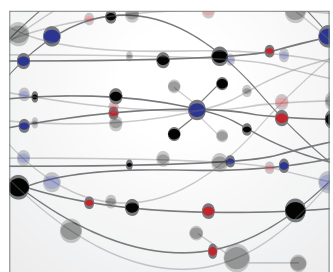

\section{The Scientific} World Journal
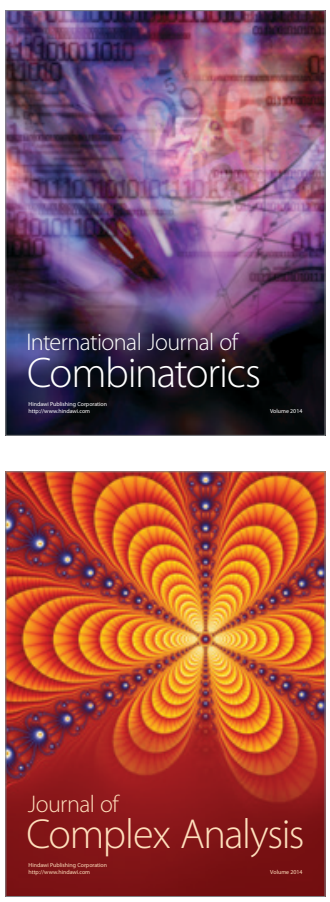

International Journal of

Mathematics and

Mathematical

Sciences
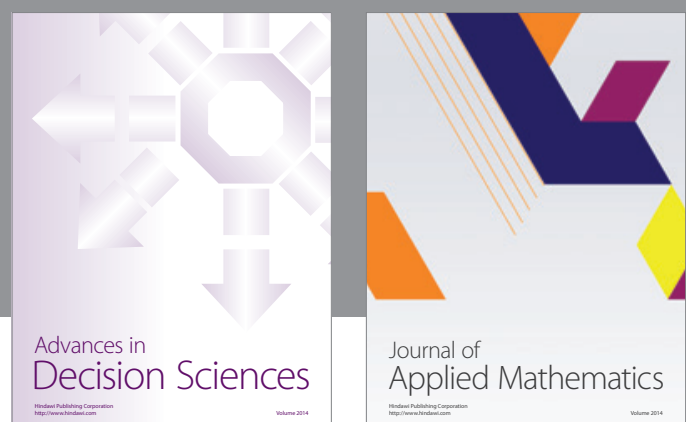

Journal of

Applied Mathematics
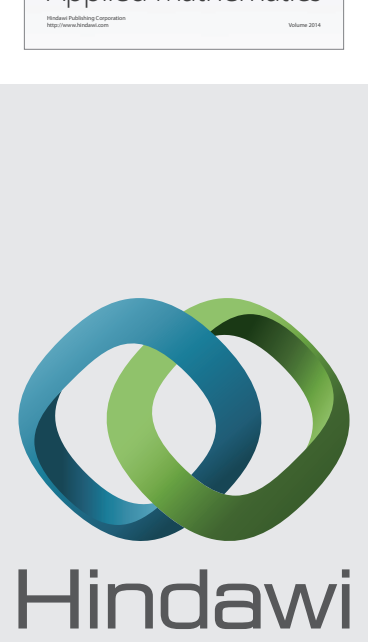

Submit your manuscripts at http://www.hindawi.com
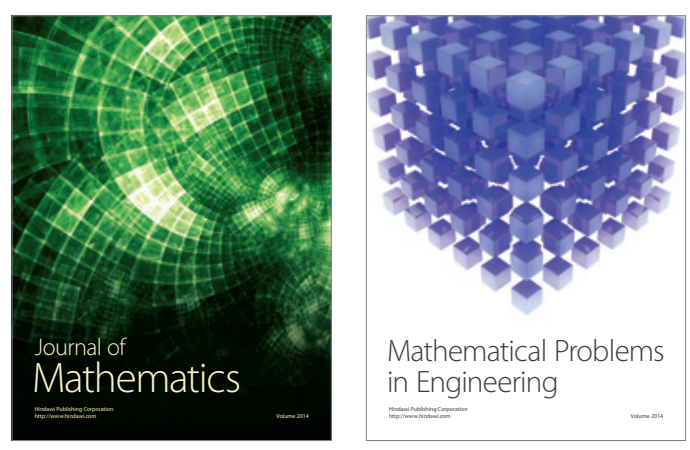

Mathematical Problems in Engineering
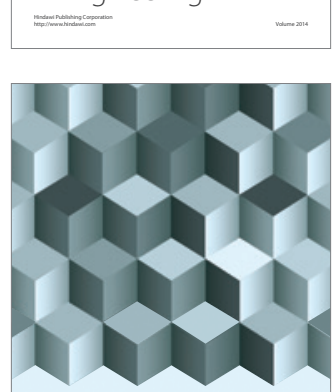

Journal of

Function Spaces
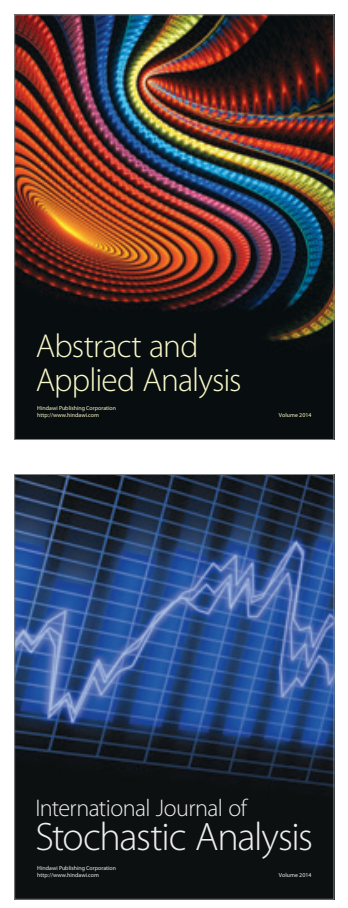

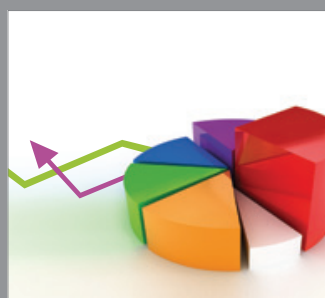

ournal of

Probability and Statistics

Promensencen
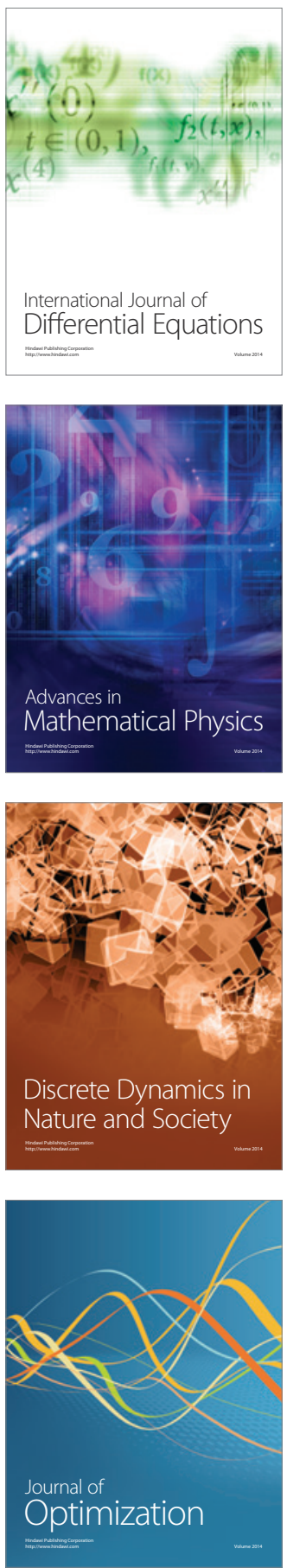